\title{
Determinación in situ del contacto de redes de arrastre de fondo
}

\author{
Teófilo Melo, Dante Queirolo, Carlos Hurtado \& Erick Gaete \\ Escuela de Ciencias del Mar, Pontificia Universidad Católica de Valparaíso \\ Casilla 1020, Valparaíso, Chile
}

RESUMEN. Se llevaron a cabo experiencias tendientes a determinar el momento en que las redes de arrastre utilizadas en la pesquería de crustáceos demersales tienen contacto efectivo con el fondo. Lo anterior permite definir en forma exacta la duración efectiva del lance, siendo este parámetro esencial para las estimaciones de abundancia llevadas a cabo por el método de área de barrido. Este documento describe los resultados obtenidos al incorporar un sensor de contacto que registra su ángulo de inclinación al ser conectado al borlón de la red de arrastre, permitiendo el monitoreo continuo de su desempeño sobre el fondo. Las mediciones de la duración del lance con dicho sensor de contacto se comparan con el método estándar utilizado, discutiéndose sus ventajas con fines de estimación de abundancia.

Palabras clave: borlón, arrastre de fondo, crustáceos, desempeño de la red, duración de lance, monitoreo de la red.

\section{In situ determination of bottom trawl gear contact}

\begin{abstract}
Tests were carried out in an attempt to determinine the moment at which the trawl gear utilized in demersal crustacean fisheries came into effective contact with the bottom. Results of the determination permit clear definition of effective tow duration, as this is an essential parameter for estimations of abundance carried out by the swept-area method. This paper presents the results obtained using a contact sensor that registers tilt angle of the footrope, permitting continuous monitoring of the net performance on the seafloor. The measurements of the tow duration with the contact sensor are compared with the method typically used, and a discussion is presented of the advantages of the new method for obtaining estimations of abundance.
\end{abstract}

Key words: footrope, bottom trawling, crustacea, net performance, tow duration, net monitoring.

\section{INTRODUCCIÓN}

Las redes de arrastre de fondo se utilizan a nivel mundial para la captura de una amplia diversidad de especies que habitan en estrecha relación con el fondo marino, existiendo gran variedad de diseños en función al comportamiento específico de cada recurso objetivo. En muchos casos la eficiencia de estas redes está en directa relación con su capacidad de operación, específicamente sobre aquellas especies que sólo pueden ser capturadas cuando el

Autor corresponsal: Teófilo Melo (tecpes@ucv.cl) borlón se encuentra en contacto con el fondo marino (Somerton \& Otto, 1999). En este sentido, Dickson (1993) explica que el escape de peces bajo el borlón puede tener un importante efecto en la eficiencia de las redes de arrastre, razón por la cual el contacto de la red con el fondo podría eventualmente afectar las estimaciones de abundancia (Wathne, 1977).

Diversos autores, tales como Rose \& Nunnallee (1998), Somerton \& Munro (2001) y Munro \& Somerton (2002) han estudiado la eficiencia de re- 
des de arrastre, principalmente orientada al efecto sobre las tasas de captura y las estimaciones de abundancia de peces. En este último caso, la principal fuente de incertidumbre en la estimación del área barrida en cruceros de evaluación corresponde a la velocidad de arrastre y a la duración efectiva del lance (Engas et al., 2001).

Una forma de mejorar las estimaciones de abundancia está asociada a la obtención de más y mejor información operacional de los lances, incrementando la precisión de los estimados de abertura de la red (Engas \& Godo, 1986; Koeller, 1991; Rose, 1993) y la longitud efectiva del área de barrido (West $\&$ Wallace, 2000). Con este último fin, Somerton \& Weinberg (2001) evaluaron el efecto que produce la velocidad de arrastre en el contacto de la red sobre el fondo, específicamente del borlón. De este modo, ellos pudieron inferir sobre la velocidad adecuada para realizar los cruceros de evaluación de stock llevados a cabo por el Alaska Fisheries Science Center (AFSC). En este sentido, Weinberg et al. (2002a) registraron el levantamiento del borlón desde el fondo asociado a cambios en la velocidad de arrastre, determinando la influencia de esta variable sobre la eficiencia de captura del arte de pesca.

La capacidad para estimar directamente la distancia de arrastre con sensores es una valiosa herramienta para la interpretación de experiencias tendientes a evaluar tasas de captura y estimación de biomasa de los recursos (Godo \& Engas, 1989; Engas et al., 1997). En este sentido, es factible identificar diversos métodos comúnmente utilizados a nivel mundial, que se relacionan con dos fuentes básicas para la obtención de información, clasificándose como indirectos y directos. Los métodos indirectos permiten obtener información a partir de sensores de profundidad en la red, de configuración de la boca de la red y de tiempos operacionales asociados al calado del arte. Los directos, en tanto, obtienen la información a partir de sensores de contacto de fondo ubicados en el borlón de la red, por lo cual son considerados como más eficientes (Engas et al., 2001).

Con respecto a las fuentes indirectas, Somerton $\&$ Munro (2001) señalan que el contacto con el fondo puede ser extrapolado a partir de la información de sensores de profundidad ubicados en la relinga, mientras que Kingsley et al. (2002) utilizan un netsonda (ojo de red) montado en la relinga para definir el inicio de los lances de evaluación, el cual entrega información relativa a la configuración de la boca de la red, específicamente su altura y abertura. No obstante lo anterior, Engas et al. (2001) y Kingsley et al. (2002) reconocen que estos procedimientos utilizados en las evaluaciones para determinar el inicio del arrastre pueden eventualmente subestimar la duración del lance, lo cual se puede explicar por el tiempo que tarda el arte en estabilizarse para su operación. Otra forma indirecta de definir el inicio del lance corresponde a la utilizada en evaluaciones directas efectuadas en Chile de camarón nailon (Heterocarpus reedi), langostino amarillo (Cervimunida johni) y langostino colorado (Pleuroncodes monodon), las cuales utilizan como procedimiento estándar el momento en que se frena la salida de los cables del winche principal (Acuña et al., 2002; Arana et al., 2003).

En cuanto a las fuentes directas, éstas se basan en la utilización de sensores de contacto del borlón, lo cual permite obtener información sistemática del desempeño de la red desde el instante en que ésta toca fondo hasta el momento en que se inicia el virado. Los sensores de contacto pueden ser clasificados en función a su principio de operación, los que corresponden básicamente a dos tipos: a) celdas de estiramiento y b) sensores de ángulo. Las celdas de estiramiento corresponden a indicadores que son comúnmente empleados como sensores de captura, los cuales permiten establecer si ésta se encuentra o no estirada (tensa) de acuerdo a la fuerza que se ejerce en sus respectivos extremos, registrando sólo una variable dicotómica (Engas et al., 2001). Los sensores de ángulo en tanto, basan su principio en la posición relativa del borlón respecto al fondo mediante el uso de un inclinómetro, permitiendo el registro continuo de esta variable. Estos últimos, denominados BCS (Bottom Contact Sensor) han sido satisfactoriamente utilizados por Munro \& Somerton (2002), Somerton \& Weinberg (2001), Weinberg et al. (2002b) y Honda et al. (2003), en sus respectivas experiencias realizadas tanto en peces demersales, como crustáceos y moluscos.

\section{MATERIALES Y MÉTODOS}

Las experiencias se llevaron a cabo en julio y octubre de 2003 frente a la costa de la zona central de Chile $\left(29^{\circ} 10^{\prime}-34^{\circ} 42^{\prime} \mathrm{S}\right)$, en fondos arenosos entre 250 y $395 \mathrm{~m}$ de profundidad. Los lances fueron realizados por dos embarcaciones comerciales que operan en las pesquerías de camarón nailon, langostino amarillo y langostino colorado, las cuales poseen 
capacidades instaladas de motor de 425 y 325 HP. Las redes utilizadas por las naves poseen similares características de diseño, armado y construcción, siendo ambas de 25,4 $\mathrm{m}$ de longitud de relinga. Los portalones en tanto, corresponden al tipo rectangular en $\mathrm{V}$, con pesos individuales de $400 \mathrm{~kg}$ y $260 \mathrm{~kg}$ para la primera y segunda embarcación respectivamente (Tabla 1).

Se realizaron 9 lances de arrastre comerciales, en los cuales se registró el tiempo de frenado del winche y de inicio del virado, correspondiendo este período a la duración nominal del lance y que tradicionalmente ha sido empleado en la pesquería y en las evaluaciones directas por el método de área barrida. En este sentido, la duración nominal del lance fue comparada con respecto a la duración efectiva del lance, que se define como el tiempo que transcurre entre el primer contacto del borlón con el fondo hasta que se inicia el virado del arte.

Para determinar el momento en el cual la red inició su operación efectiva, se empleó un sensor de contacto de fondo (BCS), el cual mide el ángulo por medio de un inclinómetro de péndulo, almacenando la información en un capturador interno de datos (Onset Computer Stow Away®). Paralelamente, el BCS permitió registrar la hora asociada a cada ángulo registrado. Los ángulos y la hora de cada registro fueron traspasados a un computador personal mediante el uso de un equipo de transfe-

Tabla 1. Características de las embarcaciones participantes en el estudio y sus artes de pesca (BHP: potencia al freno, TRG: tonelaje de registro grueso).

Table 1. Vessels characteristics employed in the research and theirs trawl gears (BHP: break horse power, TRG: gross tonnage registration).

\begin{tabular}{|lcc|}
\hline & $\begin{array}{c}\text { Embarcación } \\
\mathbf{1}\end{array}$ & $\begin{array}{c}\text { Embarcación } \\
\mathbf{2}\end{array}$ \\
\hline Eslora (m) & 21,0 & 14,9 \\
BHP (hp) & 425 & 325 \\
TRG & 85,9 & 32,1 \\
Peso portalones (kg) & 400 & 260 \\
Longitud relinga (m) & 25,4 & 25,4 \\
Longitud borlón (m) & 28,6 & 27,8 \\
Longitud red (m) & 34,5 & 29,6 \\
\hline
\end{tabular}

rencia de datos (Onset Optic Shuttle®), el cual permitió descargar la información y programar los intervalos de registro del BCS. Para las experiencias realizadas, se programó el BCS a intervalos de 5 seg.

El sensor de contacto permite registrar ángulos entre $0^{\circ}$ y $90^{\circ}$, donde $0^{\circ}$ representa su posición horizontal, mientras que $90^{\circ}$ su posición vertical. En este sentido, la posición horizontal representará entonces la situación deseada de contacto de la red durante cada lance de arrastre. La transición de los registros hacia valores menores de ángulo (idealmente tendientes a $0^{\circ}$ ) definió el contacto de la red con el fondo.

\section{RESULTADOS}

En los lances de arrastre realizados durante el estudio, la embarcación 1 operó en fondos entre 305 y $395 \mathrm{~m}$ de profundidad, empleando relaciones cable/ profundidad de 2,2-2,3:1, siendo su velocidad de remolque de 2 a 2,1 nudos. Por su parte, la embarcación 2 operó entre los 250 y $350 \mathrm{~m}$ de profundidad, con relaciones cable/profundidad de 1,9 a 2,0:1, a velocidades de 1,9 a 2,0 nudos (Tabla 2).

En función al período entre el freno del winche y el inicio del virado, se observó que la duración nominal de los lances realizados por la embarcación 1 fluctuó entre 1 h 29 min y 2 h $13 \mathrm{~min}$. La embarcación 2 en cambio realizó lances de menor duración nominal, fluctuando entre 58 min y 1 h 19 min (Tabla 3).

Mediante el uso del sensor de contacto se registró el ángulo de inclinación del borlón, en función de lo cual se determinó el momento exacto en que la red de arrastre efectivamente tuvo contacto con el fondo (Fig. 1). Con esta información se determinó la duración efectiva del lance, correspondiente al tiempo que transcurrió entre la hora de contacto de la red y el inicio del virado. De esta forma, la duración efectiva de los lances realizados por la embarcación 1 fluctuó entre 1 h 20 min y 2 h 2 min, mientras que en la embarcación 2 esta fluctuación se encontró en el rango entre 43 min y 1 h 6 min (Tabla 3).

Si se considera la duración nominal del lance como valor referencial o patrón, se observó que ésta es sobrestimada, en virtud de que la duración efectiva determinada en el presente estudio fue inferior para todos los lances evaluados (Fig. 2). Esta 
sobrestimación fluctuó, en términos generales, entre 4 y 15 min (Tabla 3 ).

Al relacionar el tiempo de retardo en el contacto de la red con respecto a la profundidad de operación, se observó que la embarcación 2 presenta una tendencia proporcional, no obstante en la embarcación 1 no fue posible observar el mismo efecto (Fig. $3)$.

\section{DISCUSIÓN}

Los resultados obtenidos indican que la duración nominal del lance sobrestima el tiempo efectivo de contacto de la red. Este retardo está asociado al tiempo requerido por el arte en alcanzar la profundidad de operación, pudiendo estar influenciada por efecto de corrientes, transientes, pesos sumergidos del arte de pesca y resistencia que genera la superficie

Tabla 2. Variables operacionales durante los lances de arrastre.

Table 2. Operational variables during the tows.

\begin{tabular}{|ccccc|}
\hline $\begin{array}{c}\text { Embarcación } \\
\text { / lance }\end{array}$ & $\begin{array}{c}\text { Profundidad } \\
\text { media (m) }\end{array}$ & $\begin{array}{c}\text { Longitud del } \\
\text { cable cala (m) }\end{array}$ & $\begin{array}{c}\text { Relación cable } \\
\text { / profundidad }\end{array}$ & $\begin{array}{c}\text { Velocidad de } \\
\text { arrastre (nudos) }\end{array}$ \\
\hline 1 / L1 & 383 & 850 & $2,2: 1$ & 2,1 \\
1 / L2 & 370 & 850 & $2,3: 1$ & 2,0 \\
1 / L3 & 305 & 700 & $2,3: 1$ & 2,0 \\
1 / L4 & 395 & 900 & $2,3: 1$ & 2,1 \\
\hline 2 / L1 & 300 & 600 & $2,0: 1$ & 1,9 \\
2 / L2 & 250 & 500 & $2,0: 1$ & 2,0 \\
2 / L3 & 270 & 550 & $2,0: 1$ & 2,0 \\
2 / L4 & 350 & 650 & $1,9: 1$ & 1,9 \\
2 / L5 & 350 & 650 & $1,9: 1$ & 1,9 \\
\hline
\end{tabular}

Tabla 3. Tiempos de duración nominal y efectiva de los lances.

Table 3. Nominal and effective tow duration times.

\begin{tabular}{|c|c|c|c|c|c|c|}
\hline $\begin{array}{c}\text { Embarcación } \\
\text { / lance }\end{array}$ & $\begin{array}{l}\text { Hora } \\
\text { Freno de los } \\
\text { winches }\end{array}$ & $\begin{array}{l}\text { de: } \\
\text { Inicio del } \\
\text { virado }\end{array}$ & $\begin{array}{c}\text { Duración } \\
\text { nominal } \\
\text { del lance } \\
\text { (h:min) }\end{array}$ & $\begin{array}{c}\text { Hora de } \\
\text { contacto } \\
\text { con el fondo } \\
\text { (h:min:s) }\end{array}$ & $\begin{array}{c}\text { Duración } \\
\text { efectiva del } \\
\text { lance } \\
\text { (h:min:s) }\end{array}$ & $\begin{array}{c}\text { Sobrestimación } \\
\text { de la duración } \\
\text { del lance } \\
\text { (h:min) }\end{array}$ \\
\hline $1 / \mathrm{L} 1$ & 12:08:00 & 13:37:00 & 01:29 & $12: 16: 51$ & 01:20:09 & 00:08 \\
\hline $1 / \mathrm{L} 2$ & $14: 27: 00$ & 16:40:00 & $02: 13$ & $14: 37: 46$ & 02:02:14 & $00: 10$ \\
\hline $1 / \mathrm{L} 3$ & $17: 25: 00$ & 19:27:00 & 02:02 & $17: 34: 31$ & $01: 52: 29$ & 00:09 \\
\hline $1 / \mathrm{L} 4$ & 20:28:00 & $22: 21: 00$ & $01: 53$ & $20: 35: 41$ & $01: 45: 19$ & 00:07 \\
\hline $2 / \mathrm{L} 1$ & 09:50:10 & 11:10:00 & 01:19 & 10:03:06 & 01:06:54 & $00: 12$ \\
\hline $2 / \mathrm{L} 2$ & $11: 50: 45$ & $12: 52: 05$ & 01:01 & 11:55:01 & 00:57:04 & 00:04 \\
\hline $2 / \mathrm{L} 3$ & $13: 15: 38$ & $14: 15: 00$ & $00: 59$ & $13: 25: 26$ & $00: 49: 34$ & 00:09 \\
\hline $2 / \mathrm{L} 4$ & $14: 59: 12$ & 16:02:09 & 01:02 & $15: 10: 41$ & $00: 51: 28$ & 00:11 \\
\hline 2 / L5 & $16: 43: 11$ & $17: 42: 10$ & $00: 58$ & $16: 58: 41$ & $00: 43: 29$ & $00: 15$ \\
\hline
\end{tabular}



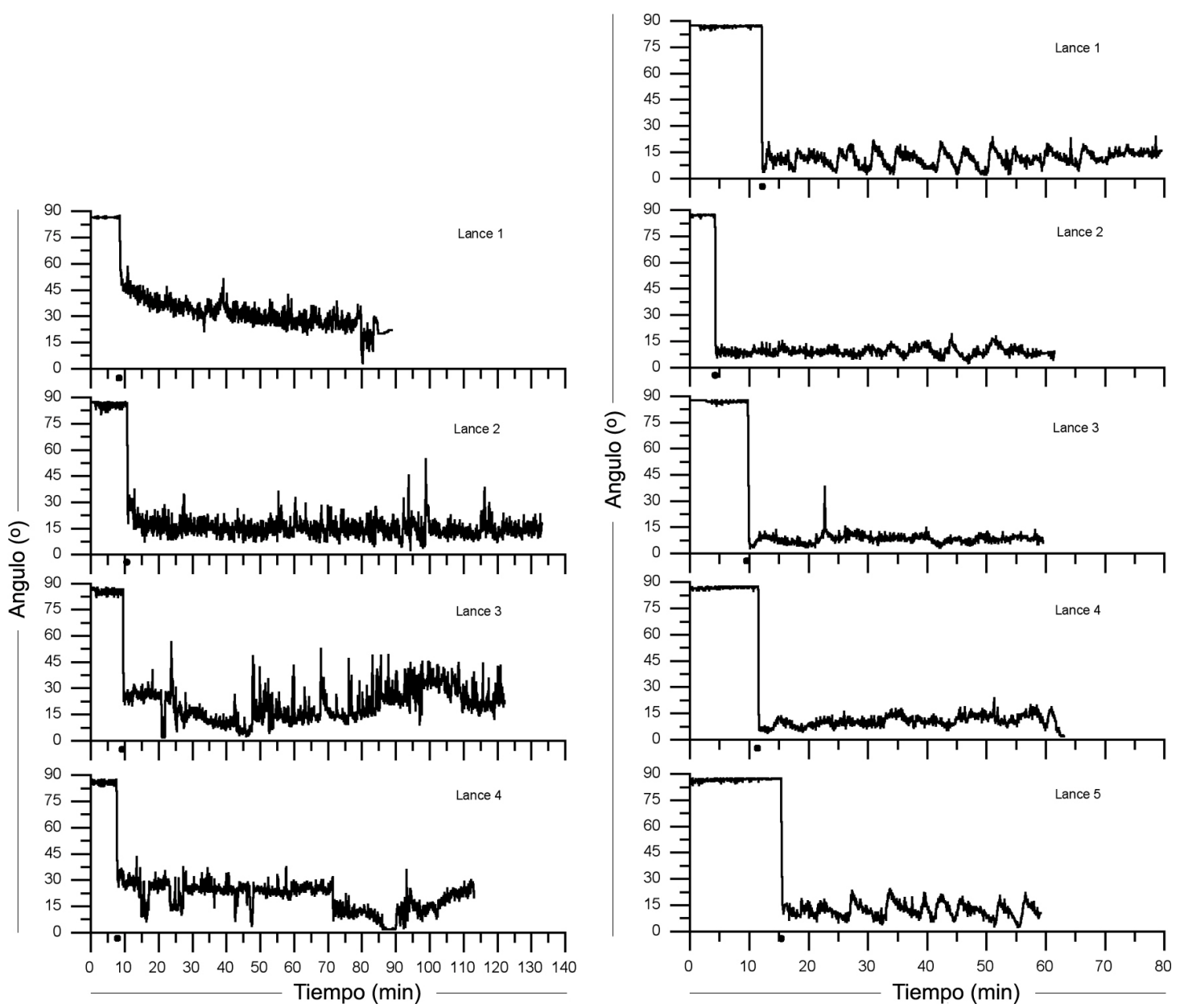

Figura 1. Mediciones de ángulo de inclinación por lance (embarcación 1 a la izquierda y embarcación 2 a la derecha).

Figure 1. Tilt angle measurements by tow (vessel 1 to left and vessel 2 to right).

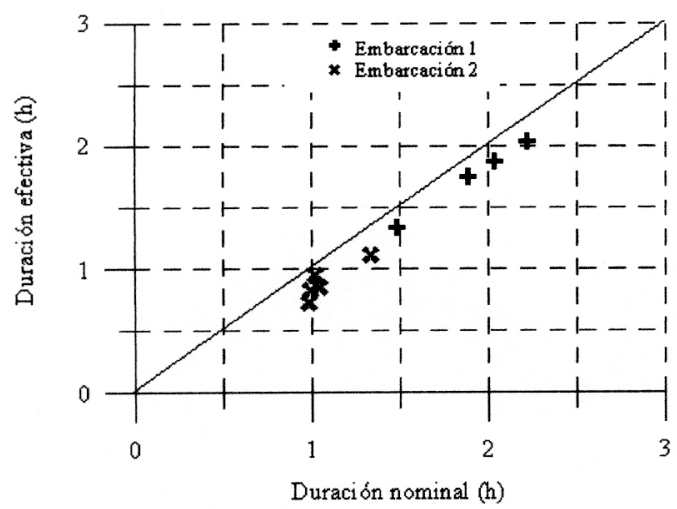

Figura 2. Comparación entre la duración nominal y efectiva del lance. La línea diagonal indica una relación 1:1.

Figure 2. Comparison between nominal and effective tow duration. Diagonal line indicate 1:1 ratio.

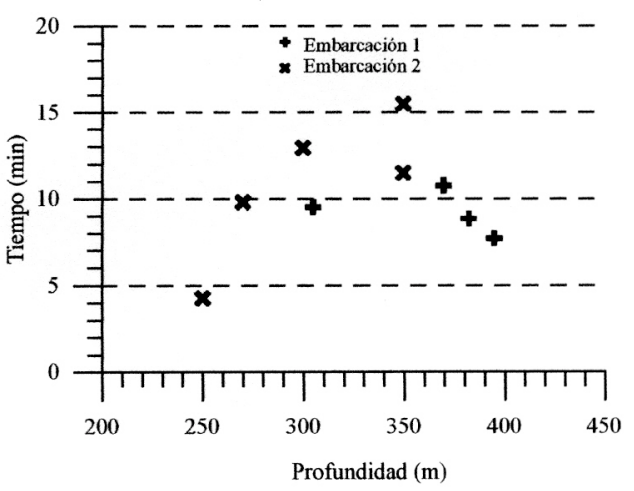

Figura 3. Tiempo sobrestimado de duración del lance en relación a la profundidad de arrastre.

Figure 3. Overestimate tow duration time in relationship with depth trawl. 
expuesta de la red durante su descenso. El hecho que no se haya observado una relación directa entre el tiempo de retardo y la profundidad, se puede explicar por la relevancia que tendrían las condiciones ambientales durante el calado del arte de pesca, especialmente la dirección de calado respecto a las corrientes, o bien por el bajo número de lances exploratorios que se llevaron a cabo. En la medida que se incorpore mayor cantidad de lances para su análisis, la evidencia debería mostrar tendencia a aumentar el tiempo de retardo en el contacto de la red con respecto a la profundidad de operación.

La incorporación de sensores de contacto de fondo en los lances comerciales, representa una valiosa herramienta para la determinación del momento en que se inicia el lance de pesca, y a partir de ello, la duración del mismo y la distancia efectiva rastreada, lo que en definitiva permitiría mejorar la estimación de los rendimientos de pesca de una embarcación. Si bien es cierto la flota que opera sobre crustáceos demersales en Chile evalúa su efectividad en función a la captura por lance, la importancia principal de estimar correctamente la distancia rastreada radica en que éste es un parámetro esencial en las estimaciones de abundancia llevadas a cabo por el método de área barrida, siendo uno de sus principales fuentes de incertidumbre (Engas $e t$ al., 2001).

El uso de sensores de contacto de fondo permite definir en forma clara el momento en que la red tiene contacto efectivo con el fondo por primera vez; no obstante, a partir de este momento algunos lances mostraron un desempeño errático del borlón, lo que quizás podría estar asociado a la topografía del terreno, a las condiciones operacionales o al estado del mar. Esto último eventualmente explicaría en mayor grado el aumento del ángulo de inclinación, debido a la transmisión de ondas o fuerzas desde la embarcación al arte de pesca. Para dilucidar esta materia, se requiere complementar con otras fuentes de información, siendo sin duda una temática de interés para mejorar aún más el tiempo efectivo de contacto.

Buscando explicación al contacto de la red con el fondo, Somerton \& Weinberg (2001) y Weinberg et al. (2002a) observaron cambios por efecto de la velocidad, mientras que Weinberg et al. (2002b) demostraron que también podrían incidir las diferentes relaciones entre la longitud del cable de cala y la profundidad de arrastre. En el caso particular de las evaluaciones directas realizadas en Chile, la velocidad de arrastre y la relación del cable de cala con respecto a la profundidad, se mantienen prácticamente constantes durante el lance de pesca, y entre lances, razón por la cual no se deberían considerar como variables principales del contacto de la red al interior de cada unidad de pesca.

Respecto del efecto de los cambios operacionales en los estimados de abundancia, Zimmermann et al. (2003) observaron en evaluaciones seriadas realizadas entre 1977 y 1998 que inusuales bajas tasas de captura probablemente estaban asociadas a un inadecuado contacto efectivo de la red en el fondo marino. Además, ellos determinaron que al mejorar el monitoreo del contacto de la red con el fondo, se obtienen mejores estimados de abundancia.

En resumen, y de acuerdo al creciente interés científico a nivel mundial, la incorporación de sensores de contacto de fondo (BCS) permite obtener información fundamental para la determinación de la duración efectiva de arrastre, y con ello reducir la incertidumbre frente a las estimaciones de biomasa realizadas por el método de área de barrido (Somerton et al., 2002). Si bien los resultados obtenidos demuestran la necesidad de profundizar en esta temática, no es factible por el momento concluir su magnitud, ni menos aún extrapolar a la totalidad de las redes de arrastre en la pesquería. En la medida que se desarrollen estudios orientados a cuantificar y evaluar el tiempo de contacto del borlón, sus resultados podrán ser incorporados en futuras evaluaciones directas de recursos pesqueros, mejorando de este modo la precisión de las estimaciones.

\section{REFERENCIAS}

Acuña, E., H. Arancibia, L. Cid, R. Alarcón, L. Cubillos \& A. Sepúlveda. 2002. Evaluación directa de camarón nailon entre la II y VIII Regiones, año 2001. Informe Final Proyecto FIP 200105. Universidad Católica del Norte. Informes Técnicos, FIP-IT/2001-05: 209 pp.

Arana, P., M. Ahumada \& A. Guerrero. 2003. Evaluación directa de camarón nailon entre la II y VIII Regiones, año 2002 (Proyecto FIP $\mathrm{N}^{\circ}$ 2002-05). Informe final. Estud. Doc., Univ. Católica Valparaíso, 05/2003: $318 \mathrm{pp}$.

Dickson, W. 1993. Estimation of the capture efficiency of trawl gear. I. Development of a theoretical model. Fish. Res., 16: 239-253. 
Engas, A. \& O.R. Godo. 1986. Influence of trawl geometry and vertical distribution of fish on sampling with bottom trawl. J. Northw. Atl. Fish. Sci., 7: 3542 .

Engas, A., E. Ona \& K. Ramberg. 2001. In situ determination of bottom trawl ground gear contact. ICES CM 2001/Q07: 9 pp.

Engas, A., R. Skeide \& C.W. West. 1997. The MultiSampler: a system for remotely opening and closing codends on a sampling trawl. Fish. Res., 29: $295-298$.

Godo, O.R. \& A. Engas. 1989. Swept area variation with depth and its influence on abundance indices of groundfish from trawl surveys. J. Northw. Atl. Fish. Sci., 9: 133-139.

Honda, N., Y. Matsushita \& K. Fujita. 2003. Development of the bottom-contact recorder for trawl. Tech. Rept. Nat. Inst. Fish. Eng., 25: 1-5.

Kingsley, M.C.S., D.M. Carlsson, P. Kanneworff \& M. Pennington. 2002. Spatial structure of the resource of Pandalus borealis and some implications for trawl survey. Fish. Res., 58: 171-183.

Koeller, P. 1991. Approaches to improving groundfish surveys abundance estimates by controlling the variability of survey gear geometric and performance. J. Northw. Atl. Fish. Sci., 11: 51-58.

Munro, P.T. \& D. Somerton. 2002. Estimating net efficiency of survey trawl for flatfishes. Fish. Res., 55: 267-279.

Rose, C. 1993. Trawl width variation and its effects on groundfish trawl survey results. $\mathrm{PhD}$ dissertation, University of Washington, Seattle, WA.

Rose, C. \& E. Nunnallee. 1998. A study of changes in groundfish trawl catching efficiency due to differences in operating width, and measures to reduce width variation. Fish. Res., 36: 139-147.
Somerton, D. 2003. Bridle efficiency of a survey trawl for flatfish: measuring the length of the bridles in contact with the bottom. Fish. Res., 60: 273-279.

Somerton, D. \& P. Munro. 2001. Bridle efficiency of a survey trawl for flatfish. US Fish. Bull., 99: 641652.

Somerton, D. \& R.S. Otto. 1999. Net efficiency of a survey trawl for snow crab, Chionoecetes opilio, and Tanner crab, C. bairdi. US Fish. Bull., 97: 617625 .

Somerton, D. \& K. Weinberg. 2001. The effect of speed through the water of footrope contact of a survey trawl. Fish. Res., 53: 17-24.

Somerton, D., R. Otto \& S. Syrjala. 2002. Can changes in tow duration on bottom trawl surveys lead to changes in CPUE and mean size? Fish. Res., 55: 63-70.

Wathne, F. 1977. Performance of trawls used in resource assessment. Mar. Fish. Rev., 39: 16-23.

Weinberg, K., D. Somerton \& P. Munro. 2002a. The effect of trawl speed on the footrope capture efficiency of a survey trawl. Fish. Res., 58: 303-313.

Weinberg, K., P. Rago, W. Wakefield \& Ch. Keith. 2002b. Estimation of tow distance and spatial heterogeneity using data from inclinometer sensors: an example using a clam survey dredge. Fish. Res., 55: 49-61.

West, C.W. \& J.R. Wallace. 2000. Measurements of distance fishing during the trawl retrieval period. ICES CM 2000/K37: 14 pp.

Zimmermann, M., M. Wilkins, K. Weinberg, R. Lauth \& F. Shaw. 2003. Influence of improved performance monitoring on the consistency of a bottom trawl survey. ICES J. Mar. Sci., 60: 818826 